\title{
Non-time Reference Gait Planning and Stability Control for Bipedal Walking
}

\author{
Xianxin Ke, Jinwu Qian and Zhenbang Gong \\ School of Mechatronics $\mathcal{E}$ Automation, Shanghai University \\ China
}

\section{Introduction}

In this increasingly aging society, the needs for robots to assist human activities in daily environments such as offices, homes, and hospitals are growing rapidly. In such environments originally designed for human beings, biped robots, which have almost the same mechanisms as a human, present many advantages than wheeled robots like obstacle avoidance capabilities for its possibility of discontinuous contact with the ground, which allows the robot to step over obstacles and climb stairs.

Biped walking robots have been in the research and development phase and biped robotics field has attracted a growing number of researchers during the last decades. Since the great success of the biped robot P2 developed by Honda in 1996(Kazuo, et al, 1998), more and more researchers focus their studies on biped robot, and have achieved many progresses. Honda has developed P3 and ASIMO afterwards. The latest version ASIMO can walk smoothly like a human being. As the most famous researcher on biped robots, Waseda

Oniversity has developed many prototypes, their latest biped robot WABIAN can even ¿ make emotional gestures (Hashimoto, et al 1998). Although Sony is a new comer in biped $\cong$ robot researches, the excellent performances of Sony Dream Robot series biped robots have ठ won good reputations, the latest robot SDR-4X can walk, dance, even stand up by itself after O falling down. As a joint research platform in Japan, HRP-2 has realized co-operations with human such as lifting a desk in cooperation with people (Tanie, et al, 1999).

Although performances of those biped robots are exciting and impressive, progresses in theoretical analysis are far behind prototype developments, a stable biped walking remains a highly demanding task for its high degrees of complexity, coupling, highly nonlinear, and unstable. Generating a desired gait for dynamic locomotion of biped robots is one of the important research areas in the study of biped robots. The simplest method to generate a d desired trajectory for a biped robot is called the inverted pendulum mode (Kajita, et al, of 1995). It is based on the assumption that all the links of the biped robot but the hip link have a negligible mass, thus the hip link representing the sole mass of the biped robot, and that 0 the biped robot contacts with the ground at a single invariant point on the sole in each cycle of the single-support phase. The trajectory obtained with the inverted pendulum mode, however, compromises locomotion stability due to the fact that the link masses, such as a foot, that are assumed to be negligible are not in fact negligible at all. An alternative method 
called the gravity-compensated inverted pendulum method generates a leg trajectory with higher stability, while keeping the most of the simplicity of the inverted pendulum mode intact (J.H. Park, 1998). A more complicated method to generate a more stable trajectory is based on the Zero Moment Point (ZMP) equation, which describes the relationship between the joint motions and the forces applied at the ground (Yamaguchi, et al , 1996). The ZMP is simply the center of pressure at the feet or foot on the ground, and the moment applied by the ground about the point is zero, as its name indicates. Yamaguchi et al. (1996) and Li et al. (1992) used trunk swing motions and trunk yaw motions, respectively, to increase the locomotion stability for arbitrary robot locomotion. However, many previous researches have assumed a predetermined ZMP trajectory. Due to the difference between the actual environment and the ideal one, or a modeling error and the impact of foot-ground, biped robots are likely to be unstable by directly using the original planned gait. In order to maintain the stability of bipedal walking, the pre-planned gait needs to be adjusted. When the robot is passing through obstacles or climbing up stairs, the adjustment of the preplanned gait may lead to the collision between the biped robot and the environment. Then the trajectory should be wholly re-planned, and the pre-planned gait becomes useless. This is the problem that conventional gait plan method encountered.

In the view of to separate the space and time, the gait of a bipedal walking can be decomposed into two parts: the geometric space path of the robot passing through, which reflect the relative movement between all moving parts of the robot; then the specific moments of the robot pass through the specific points of the geometric space path, which contain the velocities and accelerations information, and is connected to the reference of time. According to this view, we proposed a non-time reference gait planning method which can decouple the space restrictions on the path of the robot passing through and the walking stabilities. The gait planning is divided to two phases: at first, the geometric space path is determined with the consideration of the geometric constraints of the environment, using the forward trajectory of the trunk of the biped robot as the reference variable; Then the forward trajectory of the trunk is determined with the consideration of dynamic constraints including the ZMP constraint for walking stabilities. Since the geometric constraints of the environment and the ZMP constraint for walking stabilities are satisfied in different phases, the modification of the gait by the stability control will not change the geometric space path. This method simplifies the stability problem, and offline gait planning and online modification for stability can easily work together.

Gait optimization is a good way to improve the performance of bipedal walking. The optimization goal of walking stability is to make ZMP as near the center of support region as possible. This paper uses the outstanding ability of the genetic algorithm to gain a high stable gait.

Due to the difference between the actual environment and the ideal one, or a modeling error and the impact of foot-ground, online gait modification and stability control methods are needed for sure of the stable bipedal walking. When people feel about to fell down, they usually speed up the pace by instinct, and the stability is gradually restored. The changing of instantaneous velocity can restores the stability effectively. Combining the non-time reference gait planning method, a intelligent stability control strategy through modifying the instantaneous walking speed of the robot is proposed. When the robot falls forward or backward, this control strategy lets the robot accelerate or decelerate in the forward locomotion, then an additional restoring torque reversing the direction of falling will be 
added on the robot. According to the principle of non-time reference gait planning, the nontime reference variable is the only one needs to be modified in the stability control. In this paper, a fuzzy logic system is employed for the on- line correction of the non-time reference trajectory. For testify the validity of this strategy, a humanoid robot climbing upstairs is presented using the virtual prototype of humanoid robot modeling method.

This paper presents the non-time reference gait planning and stability control method for a bipedal walking. Section 2 studied the non-time reference gait planning method and the gait optimization for higher walking stability using Genetic Algorithm (GA). Section 3 built up a virtual prototype model of a humanoid robot using CAD modeling, dynamic analysis and control engineering soft wares. Section 4 studied a stability control method based on nontime reference strategy, the simulation results of a humanoid robot climbing up stairs are presented, and the conclusions and future work follow lastly.

\section{Non-time reference gait planning for bipedal walking}

\subsection{Spatial path planning}

The model of the biped robot SHUR (shown in Fig.1) used in this paper consists of two 6DOF legs and a trunk connecting them. Link the sizes and the masses of the links of the biped are given in Table 1.

\begin{tabular}{|c|c|c|c|c|c|}
\hline name & $\begin{array}{c}\text { mass } \\
(\mathrm{kg})\end{array}$ & $\begin{array}{c}\text { Ixx } \\
(\mathrm{kg} . \mathrm{m} 2)\end{array}$ & $\begin{array}{c}\text { Iyy } \\
(\mathrm{kg} . \mathrm{m} 2)\end{array}$ & $\begin{array}{c}\text { Izz } \\
(\mathrm{kg} . \mathrm{m} 2)\end{array}$ & $\begin{array}{c}\text { size } \\
(\mathrm{m})\end{array}$ \\
\hline foot & 1.17 & 0.001248 & 0.0051309 & 0.0051309 & $\begin{array}{c}\mathrm{Lf}=0.215 \\
\mathrm{wf}=0.08 \\
\mathrm{hf}=0.08\end{array}$ \\
\hline shin & 2.79 & 0.0381378 & 0.0381378 & 0.0018755 & $\mathrm{Ls}=0.4$ \\
\hline thigh & 5.94 & 0.0686441 & 0.0686441 & 0.0089843 & $\mathrm{Lt}=0.36$ \\
\hline trunk & 40.2 & 3.13895 & 2.93628 & 0.526955 & $\begin{array}{c}\mathrm{wb}=0.22 \\
\mathrm{hb}=0.91\end{array}$ \\
\hline
\end{tabular}

Table 1. Parameter of SHUR model

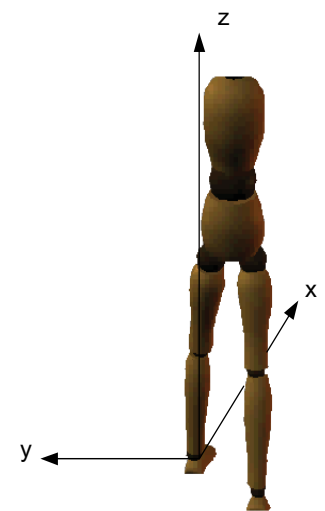

Fig. 1. Coordinate of a biped robot SHUR 
When the trunk keeps upright and the bottoms of the feet keep horizontal in gait planning, the posture of the biped robot can be decided by the positions of hip and the ankle of the swinging leg (Huang, et al, 1999). The center of mass of the robot in $\mathrm{x}$ direction $x_{\text {hip }}(t)$ plays an important role in walking stability of forward movement in which the robot tends to fall down. And $x_{\text {hip }}(t)$ is a monotonic increase function similar to the time. So, $x_{\text {hip }}(t)$ can be taken as a reference variable instead of the reference variable, time, which is usually used. Firstly, the space trajectories of the movements of the hip and the ankle of the swing leg are programmed with considerations of environmental restrictions on the robot. Then the relative movements between parts of the biped robot are fixed. Finally, the trajectory of $x_{\text {hip }}(t)$ taken time as the reference variable is planed to control the position of ZMP to realize a stable walking.

The parameters of the bipedal walking in this chapter are set:

The step length of a single step is $S_{s}=0.6 \mathrm{~m}$,

The period of a single step is $T_{s}=0.8 \mathrm{~s}$,

The maximum height the swing leg passing through is $H_{s}=0.2 \mathrm{~m}$.

\subsubsection{Spatial path planning for hip}

Because of the symmetry and periodicity of the bipedal walking, only the gait of one single step needs to be planned. Without loss of generality, it is assumed a single-step starts with the left leg to be in support and the right leg begins to swing.

It is planned that the position of the hip is located at the middle of the gap between the left foot and right foot at the moment of the support leg switched.

In a single step period,

$$
x_{\text {hip }}(t) \in\left[-\frac{S_{s}}{2}, \frac{S_{s}}{2}\right] \text {, when } t \in\left[0, \frac{T_{s}}{2}\right]
$$

Because of the symmetry and periodicity of the bipedal walking, $z_{\text {hip }}=f\left(x_{\text {hip }}\right)=z_{\text {hip }}\left(x_{\text {hip }}\right)$ is a periodic function. The period is $\frac{T}{2}=T_{s}$.

When the robot is with single support and the support leg is vertical $x_{\text {hip }}(t)=0$, the position of the hip reaches its highest point in whole cycle of bipedal walking:

$$
z_{\text {hip }}(0)=\max \left[z_{\text {hip }}\left(x_{\text {hip }}\right)\right]=l_{\text {shin }}+l_{\text {thigh }}
$$

At the moment of the supporting foot switching, the position of the hip reaches its lowest point in a period for both legs having the geometry constraints. For sure of the satisfaction of the geometry constraints at the moment of supporting foot switched, it is planned that robot retains certain flection $\delta h=0.1$.

Then, $z_{\text {hip }}\left(-\frac{S_{s}}{2}\right)=z_{\text {hip }}\left(\frac{S_{s}}{2}\right)=\min \left[z_{\text {hip }}\left(x_{\text {hip }}\right)\right]=\sqrt{\left(l_{\text {shin }}+l_{\text {thigh }}\right)^{2}-\left(\frac{S_{s}}{2}\right)^{2}}-\delta h$

The fluctuation range of the position of the hip in z-direction is: 


$$
z_{\text {hipmag }}=\max \left[z_{\text {hip }}\left(x_{\text {hip }}\right)\right]-\min \left[z_{\text {hip }}\left(x_{\text {hip }}\right)\right]=l_{\text {shin }}+l_{\text {thigh }}-\sqrt{\left(l_{\text {shin }}+l_{\text {thigh }}\right)^{2}-\left(\frac{S_{s}}{2}\right)^{2}}+\delta h
$$

The mid value of the position of the hip is:

$$
\operatorname{mid}\left[z_{\text {hip }}\left(x_{\text {hip }}\right)\right]=\min \left[z_{\text {hip }}\left(x_{\text {hip }}\right)\right]+\frac{1}{2} z_{\text {hipmag }}
$$

So, we adopt a cosine function:

$$
z_{\text {hip }}\left(x_{\text {hip }}\right)=\frac{z_{\text {hipmag }}}{2} \times \cos \left(2 \pi \frac{x_{\text {hip }}}{S_{s}}\right)+\operatorname{mid}\left[z_{\text {hip }}\left(x_{\text {hip }}\right)\right]
$$

The velocity of the hip is:

$$
\dot{z}_{h i p}=\frac{\partial z_{h i p}}{\partial t}=\frac{\partial z_{h i p}}{\partial x_{h i p}} \dot{x}_{h i p}=-\frac{\pi \mathrm{z}_{\text {hipmag }}}{2 S_{s}} \times \sin \left(2 \pi \frac{x_{h i p}}{S_{s}}\right) \dot{x}_{h i p}
$$

Thus:

$$
\dot{z}_{\text {hip }}\left(-\frac{S_{s}}{2}\right)=0, \quad \dot{z}_{h i p}\left(\frac{S_{s}}{2}\right)=0, \quad \dot{z}_{h i p}(0)=0
$$

Eq.8 means the trunk has no impact in z-direction at the moment of the supporting foot switches, which is useful to the smooth change of supporting foot. Substitute the specific parameters into the functions (Eq.6 and Eq.7), the space path and velocity of hip movement are as shown in Fig. 2.
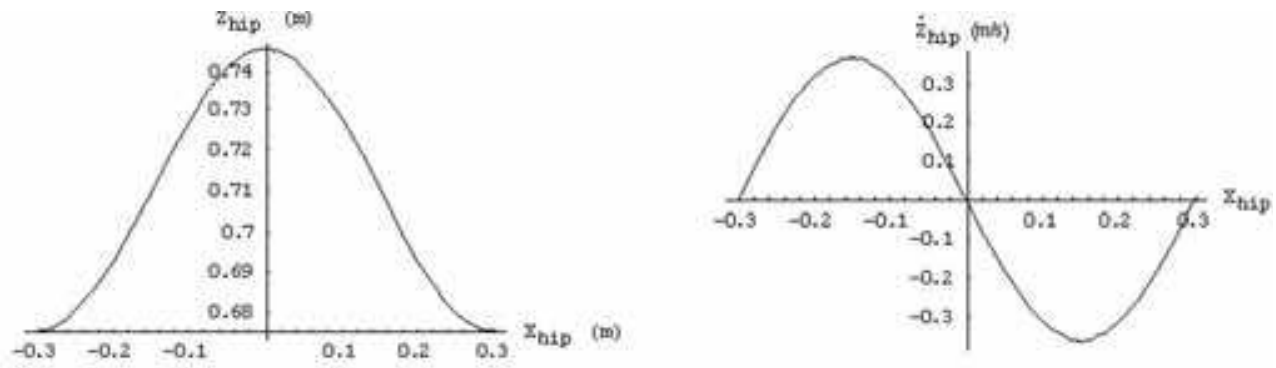

Fig. 2. Hip displacement (left) and velocity (right)

\subsubsection{Spatial Path in $\mathrm{x}$-direction ( $x_{\text {ankle }}$ ) for the Ankle of the Swing Leg}

In order to keep the process of take-off and step down smoothly, the soles of the feet are planned to be parallel to the ground during the walking process. We set $x_{\text {ankle }}$ to be a function of $x_{h i p}$ :

$$
x_{\text {ankle }}=f\left(x_{\text {hip }}\right)=x_{\text {ankle }}\left(x_{\text {hip }}\right)
$$


At the moment of the robot shifting its supporting leg, $x_{h i p}(t)= \pm S_{s} / 2$, the position of the ankle of the swing leg: $x_{\text {ankle }}= \pm S_{s}$.

When the robot stands with one foot vertically, $x_{\text {hip }}(t)=0$, the ankle of the swing leg is just above the ankle of the supported foot, that is $x_{\text {ankle }}=0$.

In order to prevent unwelcome impact during the take-off and step down process, there are constraints on velocity of the swing leg is:

$$
\dot{x}_{\text {ankle }}\left(-\frac{S_{s}}{2}\right)=\dot{x}_{\text {ankle }}\left(\frac{S_{s}}{2}\right)=0
$$

From above, we use a Sine Function (see Fig.3):

$$
x_{\text {ankle }}=S_{s} \sin \left(\frac{x_{h i p}}{S_{s}} \pi\right)
$$

Its speed is:

$$
\dot{x}_{\text {ankle }}=\frac{\partial \quad x_{\text {ankle }}}{\partial x_{\text {hip }}} \dot{x}_{\text {hip }}=\pi \cos \left(\frac{x_{\text {hip }}}{S_{s}} \pi\right) \dot{x}_{\text {hip }}
$$

Thus:

$$
\dot{x}_{\text {ankle }}\left(-\frac{S_{s}}{2}\right)=\dot{x}_{\text {ankle }}\left(\frac{S_{s}}{2}\right)=0
$$

So this path meets the requirements of no impact during supporting foot switching.
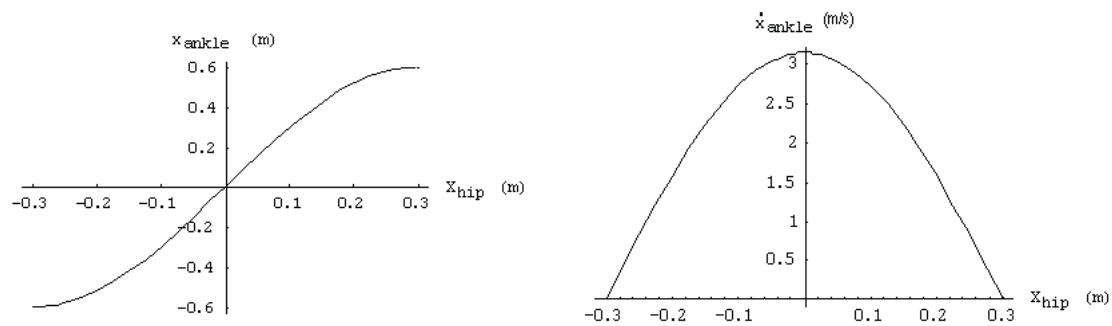

Fig. 3. Ankle displacement (left) and velocity (right) in x-axis of the Swing leg

\subsubsection{Spatial Path in z-direction ( $z_{\text {ankle }}$ ) for the Ankle of the Swing Leg}

We plan $z_{\text {ankle }}$ as a function of $x_{h i p}$ :

$$
z_{\text {ankle }}=f\left(x_{\text {hip }}\right)=z_{\text {ankle }}\left(x_{\text {hip }}\right)
$$

It follows the constraints as:

Constraints for no striking at the moment of take-off and step-down: 


$$
\dot{z}_{\text {ankle }}\left(-\frac{S_{s}}{2}\right)=\dot{z}_{\text {ankle }}\left(\frac{S_{s}}{2}\right)=0
$$

The constraint of space path:

$$
z_{\text {ankle }}\left(-\frac{S_{s}}{2}\right)=z_{\text {ankle }}\left(\frac{S_{s}}{2}\right)=0, z_{\text {ankle }}(0)=H_{s}
$$

According to the constraints above, we use a trigonometry function (see Fig.4):

$$
z_{\text {ankle }}=\frac{\mathrm{H}_{\mathrm{s}}}{2} \cos \left(2 \pi \frac{x_{\text {hip }}}{S_{s}}\right)+\frac{\mathrm{H}_{\mathrm{s}}}{2}
$$

The speed of the Ankle is:

$$
\dot{z}_{\text {ankle }}=\frac{\partial z_{\text {ankle }}}{\partial x_{\text {hip }}} \dot{x}_{\text {hip }}(t)=\frac{-\pi \mathrm{H}_{\mathrm{s}}}{S_{s}} \sin \left(2 \pi \frac{x_{\text {hip }}}{S_{s}}\right) \dot{x}_{\text {hip }}(t)
$$

Thus

$$
\dot{z}_{\text {ankle }}\left(-\frac{S_{s}}{2}\right)=0, \quad \dot{z}_{\text {ankle }}\left(\frac{S_{s}}{2}\right)=0, \quad \dot{z}_{\text {ankle }}(0)=0
$$

That is, the swing leg will not strike with the ground during take-off and step-down process.
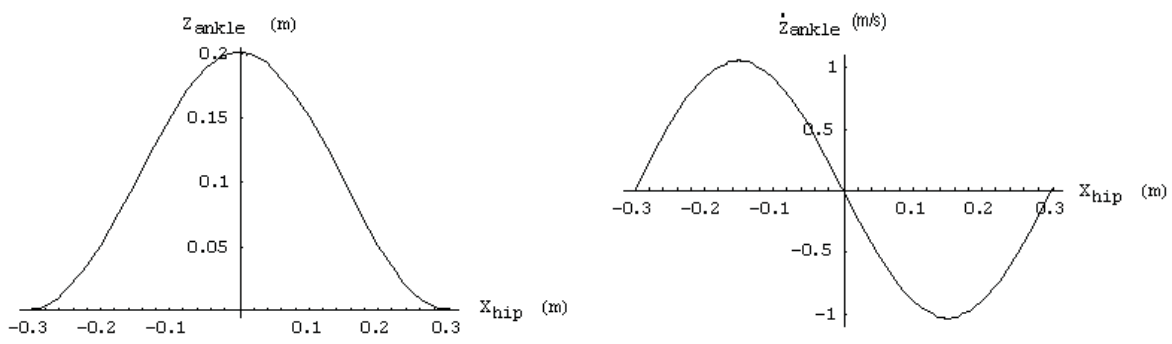

Fig. 4. Ankle displacement (left) and velocity (right) in z-axis of the Swing leg

Synthesize Eq.11 and Eq.18, we can get the spatial path of the ankle of the swing leg (Fig.5):

$$
\left\{\begin{array}{c}
x_{\text {ankle }}=S_{s} \sin \left(\frac{x_{h i p}}{S_{s}} \pi\right) \\
z_{\text {ankle }}=\frac{\mathrm{H}_{\mathrm{s}}}{2} \cos \left(2 \pi \frac{x_{\text {hip }}}{S_{s}}\right)+\frac{\mathrm{H}_{\mathrm{s}}}{2}
\end{array}\right.
$$

In which, $x_{\text {hip }}(t)$ is the referenced variable. 


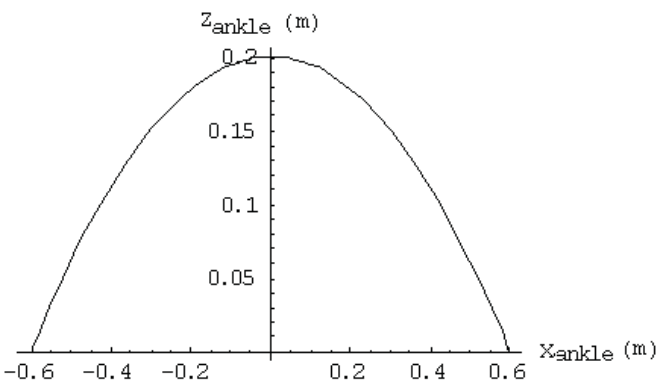

Fig. 5. Spatial path of the ankle of swing leg

\subsection{Gait planning based on ZMP stability}

Based on periodicity of bipedal walking and the symmetry of left leg and right leg, there are three equation restraints for $x_{\text {hip }}$ :

Position constraints:

$$
x_{\text {hip }}(0)=-\frac{S_{s}}{2}, x_{\text {hip }}\left(T_{s}\right)=\frac{S_{s}}{2}
$$

Velocity constraint:

$$
\dot{x}_{\text {hip }}(0)=\dot{x}_{\text {hip }}\left(T_{s}\right)
$$

As well as two inequalities constraints:

In order to save energy as well as to have the unidirectional characteristic of the time, the speed of the robot's trunk should be greater than 0 .

$$
\dot{x}_{h i p}(t)>0
$$

For sure of bipedal walking is stable, $x_{z m p}$ must be within the support region :

$$
x_{\text {heel }}<x_{z m p}<x_{\text {toe }}
$$

In order to meet these constraints at the same time, we use quintic polynomial to represent the trajectory of $x_{h i p}$.

$$
x_{\text {hip }}=a_{0}+a_{1} t+a_{2} t^{2}+a_{3} t^{3}+a_{4} t^{4}+a_{5} t^{5}
$$

Then:

$$
\begin{aligned}
& \dot{x}_{h i p}=a_{1}+2 a_{2} t+3 a_{3} t^{2}+4 a_{4} t^{3}+5 a_{5} t^{4} \\
& \ddot{x}_{h i p}=2 a_{2}+6 a_{3} t+12 a_{4} t^{2}+20 a_{5} t^{3}
\end{aligned}
$$

Substituting three constraint equations into Eq.26 and Eq.27, we get three coefficients:

$$
\begin{gathered}
x_{\text {hip }}(0)=-\frac{S_{s}}{2} \Rightarrow a_{0}=-\frac{S_{s}}{2} \\
\dot{x}_{\text {hip }}\left(T_{s}\right)=\dot{x}_{\text {hip }}(0) \Rightarrow a_{2}=-\frac{3}{2} a_{3} T_{s}-2 a_{4} T_{s}^{2}-\frac{5}{2} a_{5} T_{s}^{3}
\end{gathered}
$$




$$
x_{\text {hip }}\left(T_{s}\right)-x_{\text {hip }}(0)=S_{s} \Rightarrow a_{1}=\frac{S_{s}}{T_{s}}+\frac{1}{2} a_{3} T_{s}^{2}+a_{4} T_{s}^{3}+\frac{3}{2} a_{5} T_{s}^{4}
$$

Then we have:

$$
\begin{aligned}
x_{\text {hip }}= & -\frac{S_{s}}{2}+\left(\frac{S_{s}}{T_{s}}+\frac{1}{2} a_{3} T_{s}^{2}+a_{4} T_{s}^{3}+\frac{3}{2} a_{5} T_{s}^{4}\right) t+\left(-\frac{3}{2} a_{3} T_{s}-2 a_{4} T_{s}^{2}\right. \\
& \left.-\frac{5}{2} a_{5} T_{s}^{3}\right) t^{2}+a_{3} t^{3}+a_{4} t^{4}+a_{5} t^{5} \\
\dot{x}_{\text {hip }}= & \left(\frac{S_{s}}{T_{s}}+\frac{1}{2} a_{3} T_{s}^{2}+a_{4} T_{s}^{3}+\frac{3}{2} a_{5} T_{s}^{4}\right)+\left(-3 a_{3} T_{s}-4 a_{4} T_{s}^{2}-5 a_{5} T_{s}^{3}\right) t \\
& +3 a_{3} t^{2}+4 a_{4} t^{3}+5 a_{5} t^{4}
\end{aligned}
$$

Up to now, the question of gait planning have been changed into solving the three coefficients of the quintic polynomial under the condition of speed inequality restraints (Eq.24, Eq.25), and maximizing the stability margin of ZMP.

\subsection{Gait optimization based on walking stability using GA (Genetic Algorithm)}

\subsubsection{GA design}

Genetic Algorithm (GA) has been known to be robust for search and optimization problems. GA has been used to solve difficult problems with objective functions that do not posses properties such as continuity, differentiability, etc. It manipulates a family of possible solutions that allows the exploration of several promising areas of the solution space at the same time. GA also makes handling the constraints easy by using a penalty function vector, which converts a constrained problem to an unconstrained one. In our work, the most important constraint is the stability, which is verified by the ZMP concept. This paper applies the GA to design the gait of humanoid robot to obtain maximum stability margin, so as to enhance the robot's walking ability.

For application of optimizing using GA, there are four steps:

(1) Decide the variables which need to be optimized and all kinds of constraints;

(2) Decide the coding and decoding method for feasible solution;

(3) Definite a quantified evaluation method to individual adaptability;

(4) Design GA program, determine the operating measure with gene, and set parameters used in GA.

The parameters are set:

Population scales $M=100$,

Evolution generations $\mathrm{T}=1000$,

Overlapping probability $\mathrm{P}_{\mathrm{c}}=0.7$,

And variation probability $\mathrm{P}_{\mathrm{m}}=0.03$

The variables to be optimized are: $a_{3}, a_{4}$ and $a_{5}$

The speed constraint:

$$
\dot{x}_{\text {hip }}(t)>0, t \in\left[0, T_{s}\right]
$$




\subsubsection{The determination of the optimized goal:}

Set the projection point of the ankle of the supporting foot as the origin of the coordinate system (see Fig.1), the length from heel to the origin of the coordinate is $l_{\text {heel }}=0.08 \mathrm{~m}$, the length from the toe to the origin of coordinate is $l_{t o e}=0.135 \mathrm{~m}$, the central position of the support foot is:

$$
x_{\text {footcenter }}=\frac{l_{\text {toe }}-l_{\text {heel }}}{2}
$$

In a bipedal walking cycle, the ZMP stability in $\mathrm{x}$ direction can be expressed as:

$$
-l_{\text {heel }}<x_{z m p}<l_{\text {toe }}
$$

The index of $x_{z m p}$ offsetting the center of the support region is:

$$
S_{\text {index }}=\left|x_{z m p}-x_{\text {footcenter }}\right|
$$

The value of the index is smaller, the stable margin is bigger. Therefore the optimizing goal can be set as:

$$
\text { Object : Minimize }\left[J\left(a_{3}, a_{4}, a_{5}\right)\right]
$$

In which,

$$
J\left(a_{3}, a_{4}, a_{5}\right)=\operatorname{Max}\left[\left|x_{z m p}(t)-x_{\text {footcenter }}\right|, t \in\left[0, T_{s}\right]\right]
$$

Taking the constraints in consideration, the optimizing goal is modified as:

Object : Minimize $(J+g)$

In which,

$$
g=\left\{\begin{array}{cc}
0 & \dot{x}_{\text {hip }}>0 \\
l_{\text {foot }} & \dot{x}_{\text {hip }} \leq 0
\end{array}\right.
$$

\subsubsection{Optimized results}

By using the toolbox of MATLAB------Genetic Algorithm for Function Optimization of Christopher R.Houck, with the optimize process shown in Fig.6 and Fig.7, we get the optimized values of all variables:

The value of the optimize goal:

$$
\begin{aligned}
& a_{3}=11.1184 \\
& a_{4}=-13.9498 \\
& a_{5}=6.9642
\end{aligned}
$$

The minimum distance between $X_{z m p}$ and the support region boundary is $0.052 \mathrm{~m}$, so the stability margin is big enough. Substitute $a_{3}, a_{4}$ and $a_{5}$ into $X_{\text {hip }}$, then the planned gait is obtained (refer to Fig.8): 

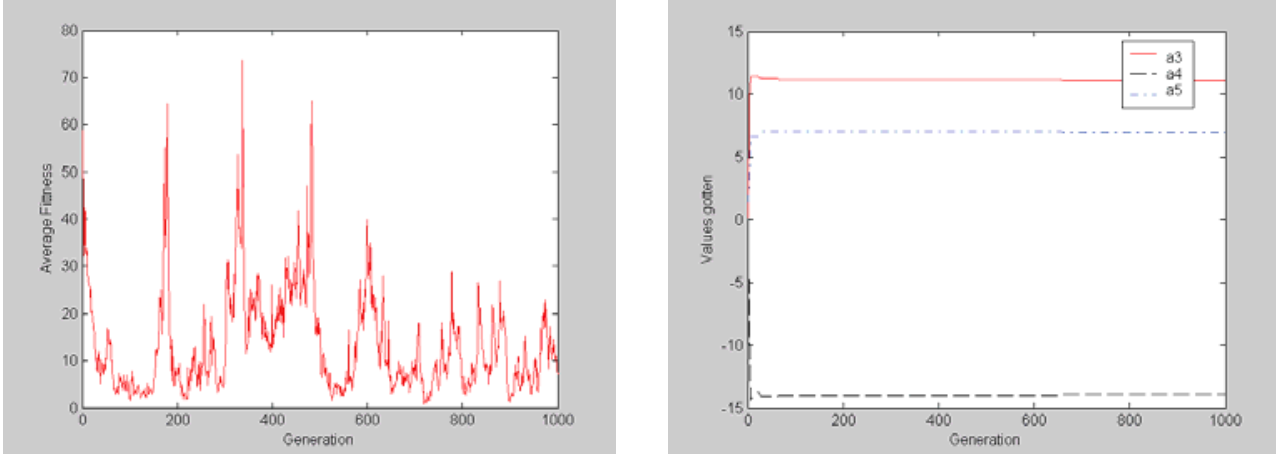

Fig. 6. Average adaptability (left) and the value of the variables (right)

Fig.9 shows that when the position of the center-of-gravity $x_{c g}$ is outside the support region, the $x_{z m p}$ of the planning gait optimized by using of Genetic Algorithm is still at the center of the support region. This optimized gait has greater stability margin, the capacity of antijamming improved during bipedal walking, and the physical feasible of the planned gait is guaranteed.

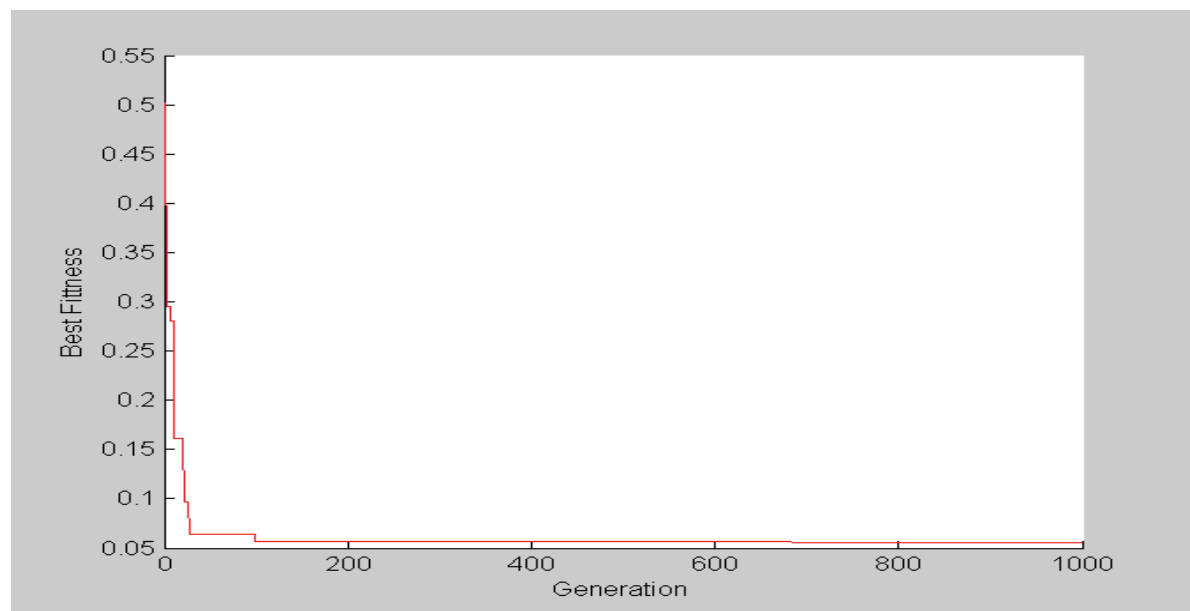

Fig. 7. Optimized adaptability
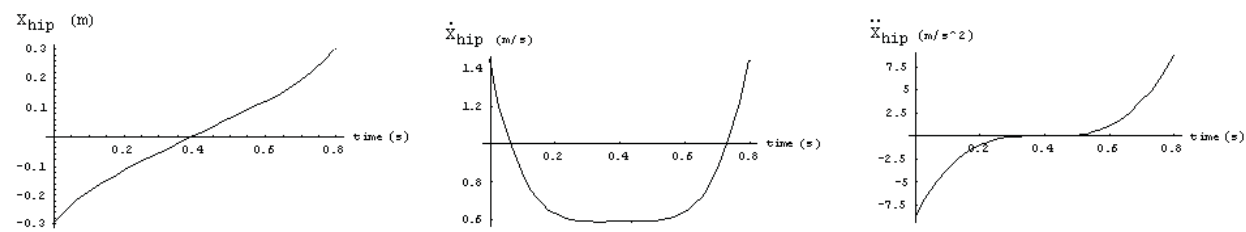

Fig. 8. Optimized Trajectories of $X_{\text {hip }}$ 


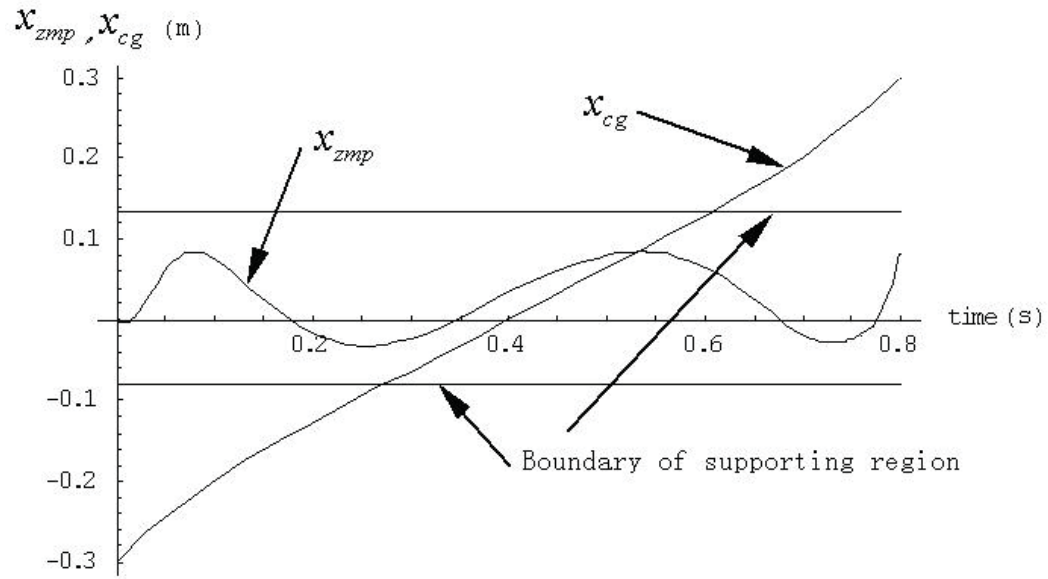

Fig. 9. Centre-of-Gravity and ZMP trajectories of the optimized gait

\section{Virtual prototype model of humanoid robot}

\subsection{Mechanical model in ADAMS}

For exactly building a virtual prototype of the humanoid robot SHUR, a various professional soft wares are used. The geometric model of the humanoid robot SHUR is built in professional three-dimensional CAD soft Pro/E, its dynamics simulation is in ADAMS soft ware, the design the robot control system is in MATLAB soft ware. Through ADAMS/Controls interface module, a real-time data channels between MATLAB and ADAMS is build, and an associated simulation is implemented.

The mechanical system model of the humanoid robot SHUR in ADAMS must include geometries, constraints, forces, torques and sensors. The procedure of building the model includes eight steps.

(1) Building part models for all parts of the humanoid robot, then assembly part models together through applying geometric constraints as the robot being at the posture of standing.

(2) Setup environment parameters of ADAMS

(3) Using the interface module of Mechanical/pro between Pro/e and ADAMS, the assembled model is imported into ADAMS;

(4) Building pairs (joint) between each adjacent links, and applying locomotion constraint.

(5) Building contact models between feet of the humanoid robot and the ground.

(6) Setting locomotion constraints at particular joints.

(7) Applying driving torques on joints relating to bipedal walking motion.

(8) Building virtual sensors to receive state information of the system 


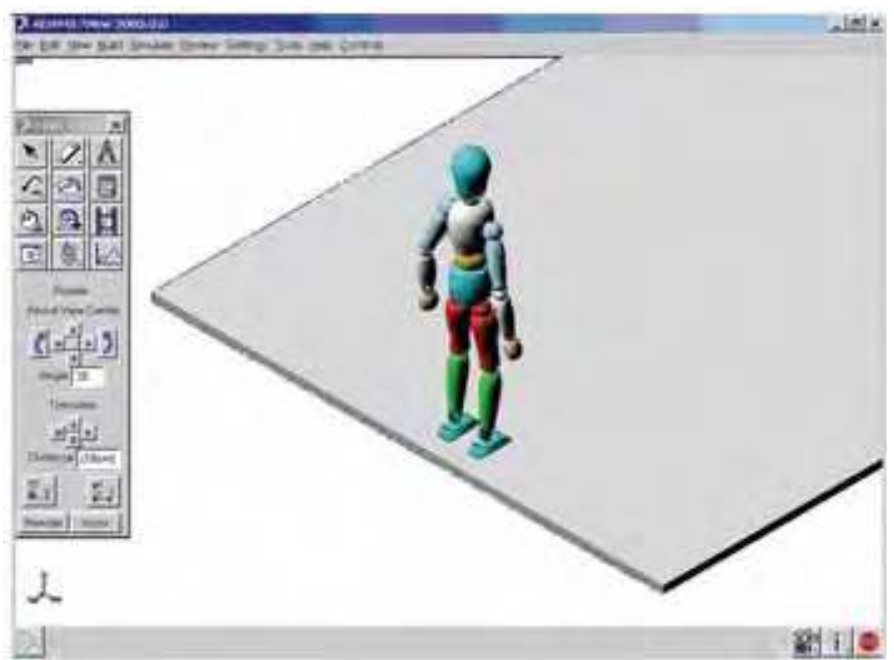

Fig. 10. Virtual prototype of humanoid robot SHUR

Fig.10 and Fig.11 show the virtual principle prototype of the humanoid robot SHUR, including 17 movable links, 16 ball hinge joints and the contact models between both feet and the ground.

\begin{tabular}{|c|c|}
\hline$=00012^{2}$ & $\begin{array}{l}\text { Bodel } \\
\text { fart }\end{array}$ \\
\hline $\begin{array}{l}\text { Tabdosen } \\
\text { i chant }\end{array}$ & part \\
\hline 1. ground & part (ground) \\
\hline + head & fate \\
\hline t hap & Part \\
\hline - lettstoot & part \\
\hline + Hetecorears & Bart \\
\hline + Lerchand & Part \\
\hline + Ieteshis & Part \\
\hline t 1efteshoulder & tare \\
\hline + lettehigh & Part \\
\hline t neels & pert \\
\hline + is the foet & part \\
\hline + righe totears & hars \\
\hline t righehand & part \\
\hline 1. Fighe phin & tart \\
\hline t righeshoulder. & Pare \\
\hline + riglasehiph & part \\
\hline Jorrt_abdowen_hlp & Spherieal joint \\
\hline Jornt_chent_abdooen & Bgharseal 2oim \\
\hline 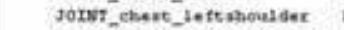 & Dpherbeat fotne \\
\hline Jornt_ebext_xight shouldat & Beharical fotme \\
\hline Jorst_hosd_nect & Apherieal Joinh \\
\hline Jorst_hap_lofethigh & Ipherseal Jo1m \\
\hline 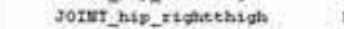 & Bpharticat Jaine \\
\hline Jorkt_lefeterears__istchand & Bspuencal foum \\
\hline JorNt_1ettshan_left toot & Iplierieal JoLx \\
\hline JoIst_1eftaboulder__eft forv & rara spherieal Joins \\
\hline sorvi_lefucheph_ieftesin & Spherical Joist \\
\hline Jorirt_neck_ebest & Spherseal Jount \\
\hline 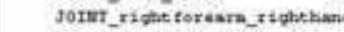 & 14 epterieal foint \\
\hline sorkt_xi phe thin righe fove & Spherieal Joine \\
\hline 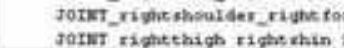 & $\begin{array}{l}\text { mears spherical Joint } \\
\text { spharical foins }\end{array}$ \\
\hline
\end{tabular}

Fig. 11. Basic components and main joints of SHUR 


\subsection{Virtual prototype system of humanoid robot SHUR}

The input and output variables of the model in ADAMS are defined. The input variables are the required control variables, that is, the driving moment of the joints. The output variable is the measuring quantity of sensors, which are the state information of the system, mainly including: angular displacement, angular velocity, and angular acceleration of each joint and the state of whole robot, such as CoG, ZMP, and inclination state of the robot and so on. MATLAB soft ware is used to build a control system block diagram of the control system of humanoid robot SHUR (Fig.12). The ADAMS mechanical system must be included in block diagram, so as to complete a closed loop system including ADAMS and control system soft MATLAB.

The simulation of the whole system is processed by using suitable control laws. The 3D solid models, kinematics, dynamic model and animation simulation of the humanoid robot are supplied by ADAMS; the expected gait and the control algorithm are supplied by MATLAB, and the driving moment of each joint is the output of MATLAB. Through the interface provided by ADAMS/control module, MATLAB provides the control command of the driving moment of each joint to ADAMS; the latter will feedback the virtual sensor information of the system states into MATLAB, a real-time closed loop control system is completed. The result of the simulation may be displayed and saved through data, drawings and animations in ADAMS.

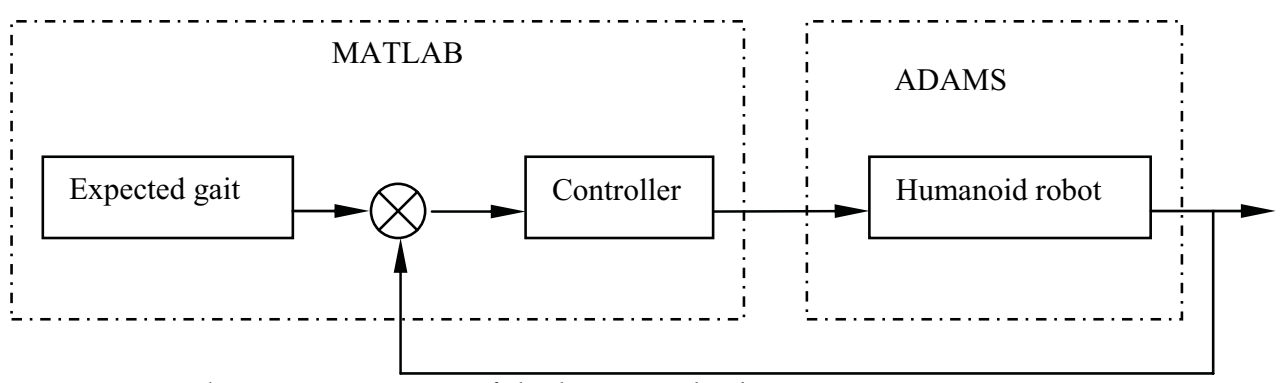

Fig. 12. Virtual prototype system of the humanoid robot SHUR

\section{Non-time reference stability control method}

\subsection{Principle of stability control through modifying the walking speed}

A biped robot may be viewed as a ballistic mechanism that intermittently interacts with its environment, the ground, through its feet. The supporting foot / ground "joint" is unilateral for there is no attractive forces, and underactuated since control inputs are absent. Formally, unilateral and underactuation are the inherent characteristics of biped walking, leading to the instability problem, especially un-expected falling down around the edge of the support foot. This stability problem can be measured by ZMP and or be measured by a more visual index of the degree of inclination of the robot. Almost every humanoid robot has installed the sensors like gyroscope to measure the degree of inclination of the robot. A virtual gyroscope is installed on the virtual prototype of the humanoid robot SHUR to measure the inclination angle of the posture of the upper body. This inclination angle is the object of the stability control in our research. At the same time, the inclination angle is also used as the feedback information of the close-loop stability control system. 
To simplify the architecture of the controller, a 2-level control structure including coordination and control levels is introduced (see Fig.13).The coordination level is in charge of controlling the stability of bipedal walking. The main tasks of coordination level include gait planning; coordinate the movements of every part and giving command to the control level. The control levels receive the command from the coordination level and realize the trajectory tracking controls of every joint of the humanoid robot.

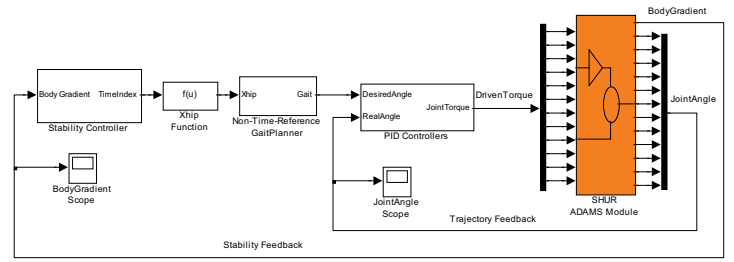

Fig. 13. Walking control system in MATLAB

The ZMP trajectory can be easily planned to be located in the valid support region at the phase of off-line gait planning. But in actual walk, there are the differences between the actual environment and the ideal one, or a modeling error, the impact of foot-ground, as well as external interference, which cause the real ZMP trajectory differ from the predesigned one. If this difference is in open-loop state, the robot walks directly using the original planned gait, the stability may be broken down, the pre-planned gait can not be realized, so, it is necessary to correct the gait path on-line.

When people feel about to fell down, they usually quickens the pace to reduce the overturning moment and gradually restores to stable walk. The changing of instantaneous velocity can restores the stability effectively. Restoring the walk stability by changing instantaneous walk speed nearly has become a person's instinct of responding, which is gradually gained through the practices of bipedal walking. This paper uses the same method of human beings to achieve a stable walk. When the robot falls forward or backward, the strategy lets the robot accelerate or decelerate in the forward locomotion, then an additional restoring torque reversing the direction of falling will be added on the robot.

Does not lose the generality, taking robot falling forward around the edge of the toe of the support foot as an example, this paper uses an on-line correct method to accelerate the forward locomotion of the robot to restore the walking stability. When the robot is accelerated forward, there is an additional forward acceleration

$$
\Delta \ddot{x}_{\text {hip }}>0
$$

The robot receives a backward additional force

$$
\Delta F_{x}=-m \Delta \ddot{x}_{h i p}<0
$$

The backward additional force will produce a restoring moment relating to the support foot, which is opposite to the direction of falling.

$$
\Delta M_{y}=\Delta F_{x} \cdot h_{c m b}
$$

In which, $h_{c m b}$ is the height of the center of the mass of the trunk to the ground. The additional moment $\Delta M_{y}$ is helpful for ZMP to restore to the center of the support region. 
For falling forward, this strategy of accelerating forward will also let the swing leg touch the ground sooner than original planning, so the robot will get a new support, the falling forward trend will be stopped.

When we off-line planned the robot space path, we had already considered the robot walking environmental factors like obstacles or the topographic factor like staircase specification, the ground gradient and so on. Therefore, the online gait modification had better not to change the robot space path which be planned off-line. Referring to the nontime gait planning principle, the non-time reference variable is the only one needs to be modified in the stability control. So the online modify algorithm can be realized easily based on offline gait planning, and the space path of the robot passing through remains unchanged.

Applying non-time gait planning algorithm, the whole gait-planning phase is divided into two phases, (1) planning the space walking path: Taking the forward locomotion of upperbody as the reference variable, considering the constraint of the environment, the walking path of a robot without collision with other objects is designed, thus the relating locomotion of the parts of the robot is obtained; (2) planning the trajectory of the non-time reference variable: according the constraint of ZMP stability, design the forward locomotion of upperbody. By changing the forward locomotion of upper-body. $x_{h i p}(t)$, the dynamics characteristic can be changed to satisfy the walking stability condition while the space walking path maintains unchanged.

The trajectory of the upper body of the robot in forward direction is:

$$
x_{\text {hip }}=a_{0}+a_{1} t+a_{2} t^{2}+a_{3} t^{3}+a_{4} t^{4}+a_{5} t^{5}
$$

Applying non-time reference principle to the trajectory of the upper body in forward direction, we replace the variable (time t) in the quintic polynomial with a non-time parameter (time index time $_{\text {index }}$ ), The time index time $_{\text {index }}$ is a function of the time: time $_{\text {index }}=f(t)$. In control and simulation, the time index time $_{\text {index }}$ is a discrete time series basically separated by the sample time interval (in virtual prototype, it is the time step length of simulation, SimTimeStep ).

$$
\text { time }_{\text {index }}{ }^{n+1}=\text { time }_{\text {index }}{ }^{n}+\text { SimTimeStep }+\Delta \text { time }_{\text {index }}{ }^{n+1}
$$

In which, $\Delta$ time $e_{\text {index }}$ is the time index correction decided according to the stability states of the robot.

For guarantee the robot will not stop or go back because of the gait correction, the time index should satisfy:

So the inequality must be satisfied:

$$
\text { time }_{\text {index }}{ }^{n+1}>\text { time }_{\text {index }}{ }^{n+1}
$$

$$
\Delta \text { time }_{\text {index }}>- \text { SimTimeStep }
$$

In certain scope, if $\Delta$ time $e_{\text {index }}>0$, the forward walks speed is accelerated compare to the offline planned one, otherwise decelerated. As shown in Fig.14, using a fuzzy controller to determinate the time index $\Delta$ time $_{\text {index }}$ according to the upper body gradient which corresponding to the states of stability. 


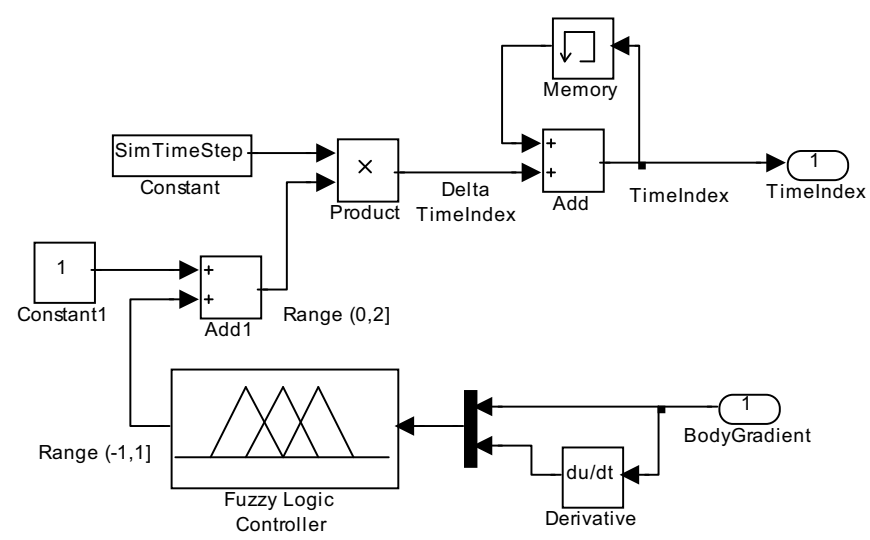

Fig. 14. Time index modification system using fuzzy controller

Regarding to general gait planning methods, the planned gaits in joint space are:

$$
\theta_{i}=f(t) \quad i=1,2, \ldots n J o i n t
$$

If we replace the variable $t$ in Eq.50 with the time index time $_{\text {index }}$, we can also correct the gaits online using the non-time reference stability control algorithm, according to the stability states of the bipedal walking. The relative motion paths of the joints remain unchanged after the gait correction, which means the robot space motion paths remain unchanged.

\subsection{Design of Fuzzy Controller}

Fuzzy control is a combination of fuzzy logic and control technology, and has advantages to control the systems which are indeterminate, highly nonlinearity and complex. So we adopt a fuzzy controller to achieve the nonlinearity mapping between the BodyGradient and the increment of the time index.

A fuzzy controller shown in Fig.16 is built by using the fuzzy controller tool box in MATLAB. The Inputs of the fuzzy controller are BodyGradient and GradientRate, and the output is Coefficient. Fig.17 shows the range of values and membership functions of these input and output variables.

The variable BodyGradient has three ranges: Forward, Okey and Backward. The variable GradientRate has three ranges: Negative, Neglectable and Positive. The variable Coefficient is classified into five ranges: Lower, Low, NoChange, Fast, and Faster.

There are many methods to derive fuzzy rules for the biped control(Pratt, et al,1998), either from intuitive knowledge of the biped control by human walking demonstration(G.O.A. Zapata, et al,1999), or information integration(Zhou,2000). Based on intuitive balancing knowledge, nine fuzzy rules are obtained as shown in Fig.17 (left), and the relationship between the inputs and the output of the fuzzy controller is shown inFig.17 (right) 


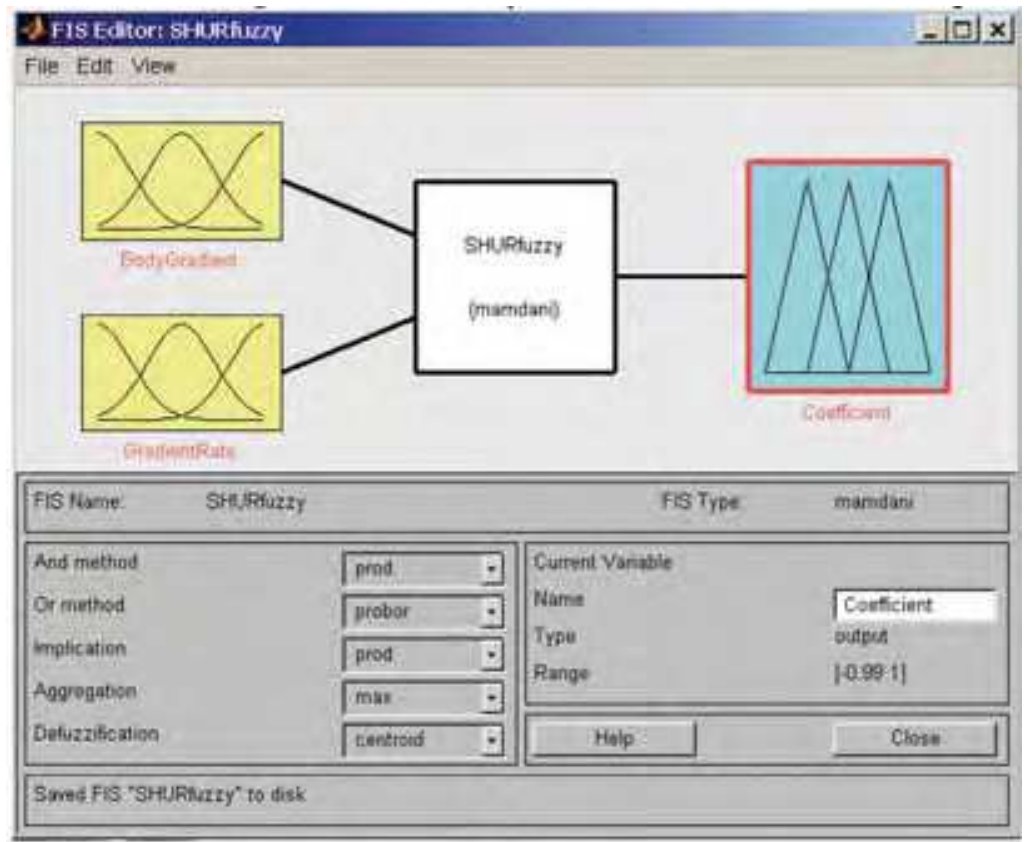

Fig. 15. Structure of the fuzzy system

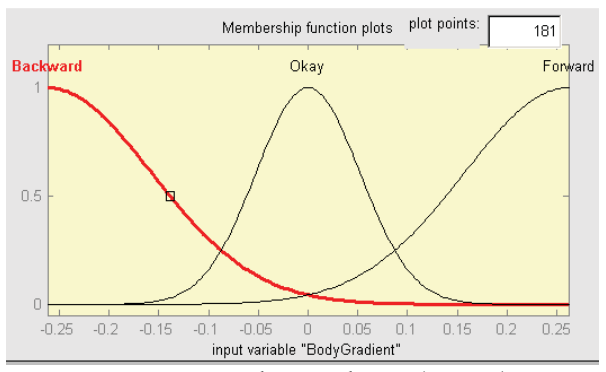

A. BodyGradient (input)

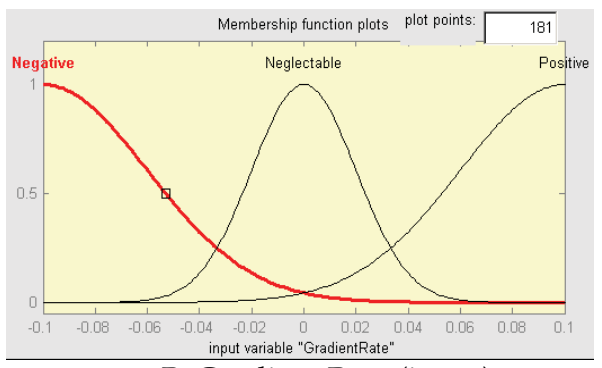

B. Gradient Rate (input)

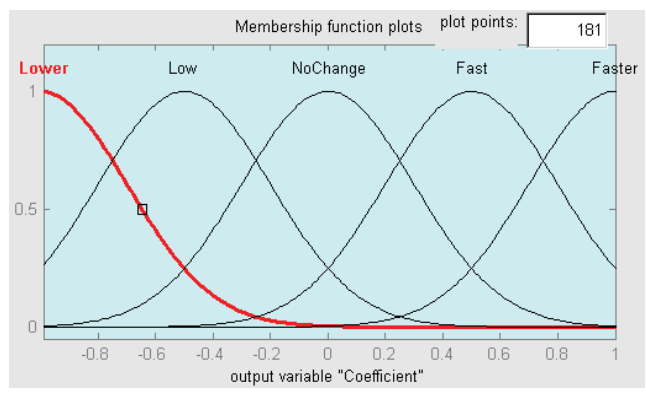

C. Time exponent modification parameter (output)

Fig. 16. Membership functions of the input and output variables of the fuzzy controller 


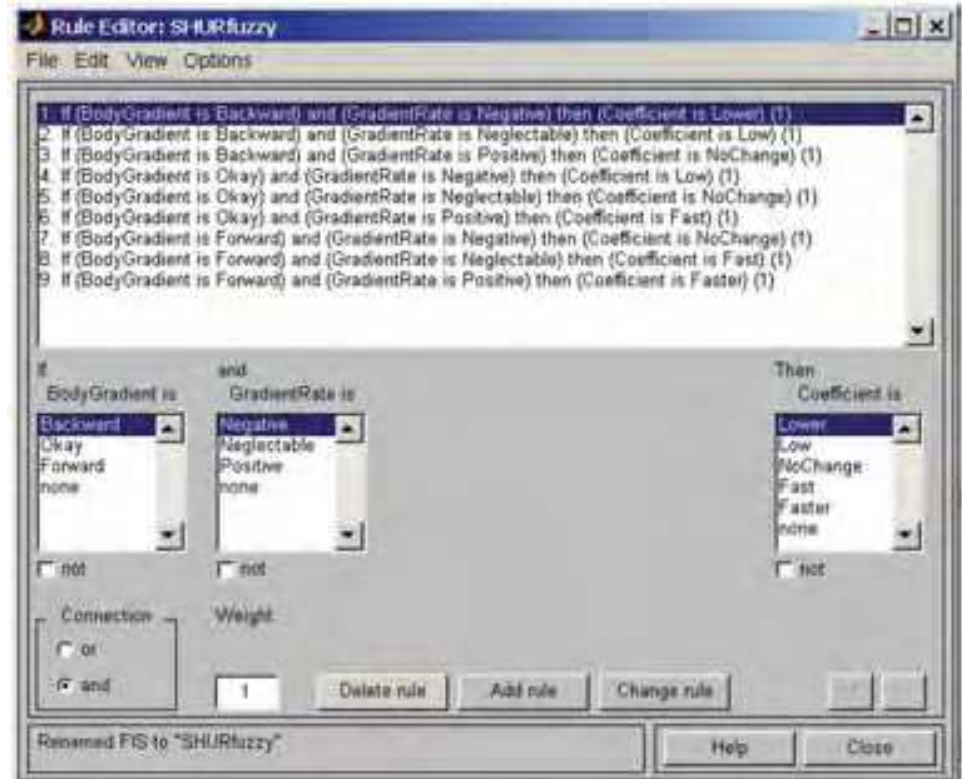

Fig. 17. Nine fuzzy rules of the fuzzy controller

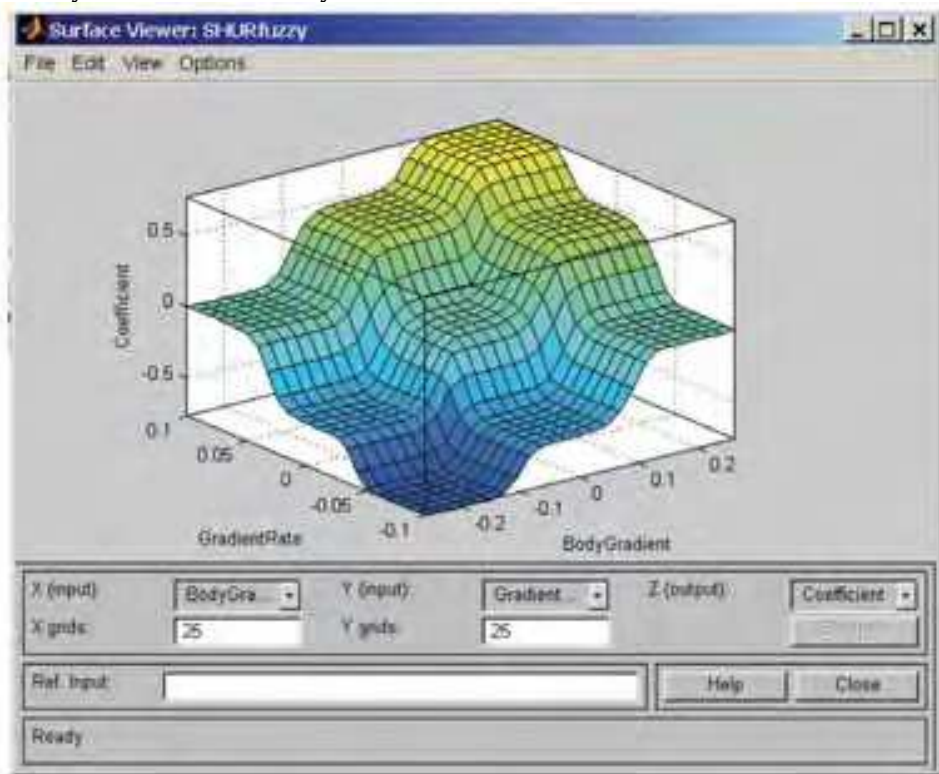

Fig. 17. Fuzzy rules (left) and the relationship between the input and output of the controller (right) 


\subsection{Simulations of climbing upstairs}

The simulation of climbing up stairs is realized by the virtual prototype of humanoid robot SHUR, using the non-time reference dynamic stability control strategies. The simulation parameters include, the height of the stair is $0.15 \mathrm{~m}$, and the depth of the stair is $0.2 \mathrm{~m}$, the period of a single step is $0.8 \mathrm{~s}$. The beginning and the ending phases of the gait have a single step period. Fig.18 shows the virtual prototype of the humanoid robot SHUR and the virtual environment including stairs. Fig.19 shows motion sequences t of climbing upstairs.

\section{Conclusions and discussions}

A non-time reference gait planning method is proposed. The usual reference variable, time, is substituted by a non-time variable in gait, so the whole gait-planning phase can be divided into two phases, (1) planning the space walking path: Taking the forward locomotion of upper-body as reference variable, considering the constraint of the environment, the walking path of a robot without collision with other objects is designed, thus the relative locomotion of the parts of the robot is obtained; (2) planning the trajectory of the non-time reference variable: according to the constraint of ZMP stability, design the forward locomotion of upper-body. The gait-planning problem is changed to the optimization problem. Using the excellent optimization and searching property of Genetic Algorithm, the gait with good stability is obtained. This non-time reference gait planning methods has advantages in passing obstacles, climbing upstairs or downstairs and other similar situation in which the walking path is specified. In the progress of stability control, the non-time reference variable is the only one need to be modified, so the online modify algorithm can be realized easily based on offline gait planning.

Combining the non-time reference gait planning method, the intelligent stability control strategy through modifying the instantaneous walking speed of the robot is proposed. When the robot falls forward or backward, the strategy lets the robot accelerate or decelerate in the forward locomotion, then an additional restoring torque reversing the direction of falling will be added on the robot. For falling forward, this strategy will also let the swing leg touch the ground sooner than original planning, so the robot will get a new support, the falling forward trend will be stopped. According to the principle of non-time reference gait planning, the non-time reference variable is the only one needs to be modified in the stability control. The incline state of the upper-body, which reflects the stability state of the robot directly, is used as the input signal of a fuzzy controller; the correction of the non-time reference trajectory is used as the output of the fuzzy controller. Then the walking speed is changed, so the gait of the robot is modified online to realize stable dynamic walking without changing the off-line planned walking space path. For testify the validity of this strategy, the humanoid robot climbing upstairs is realized using the virtual prototype of humanoid robot. 


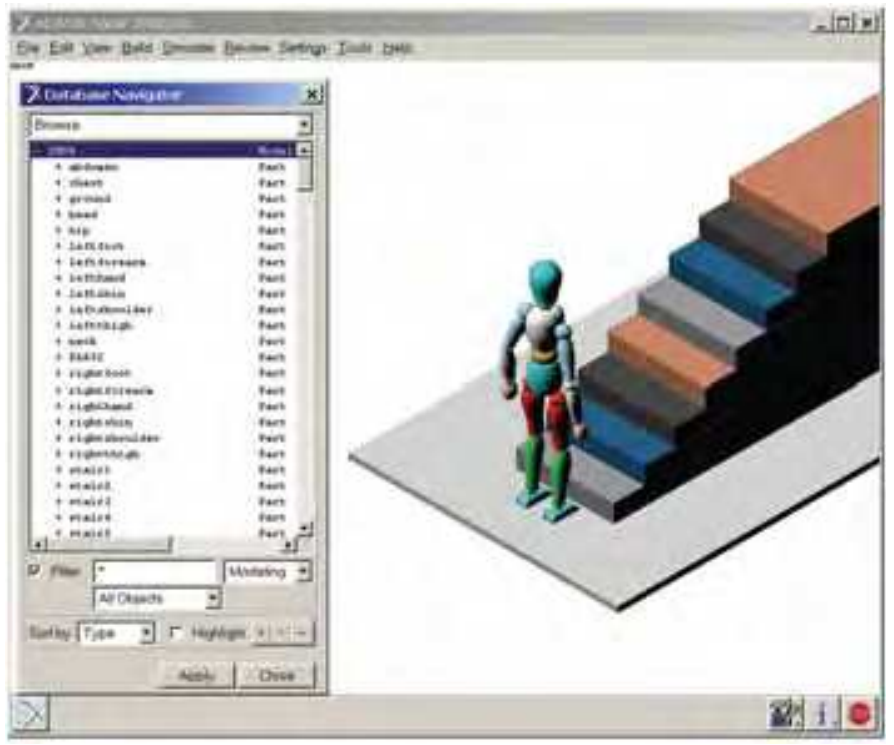

Fig. 18. Virtual prototype of a humanoid robot with virtual environment including stairs
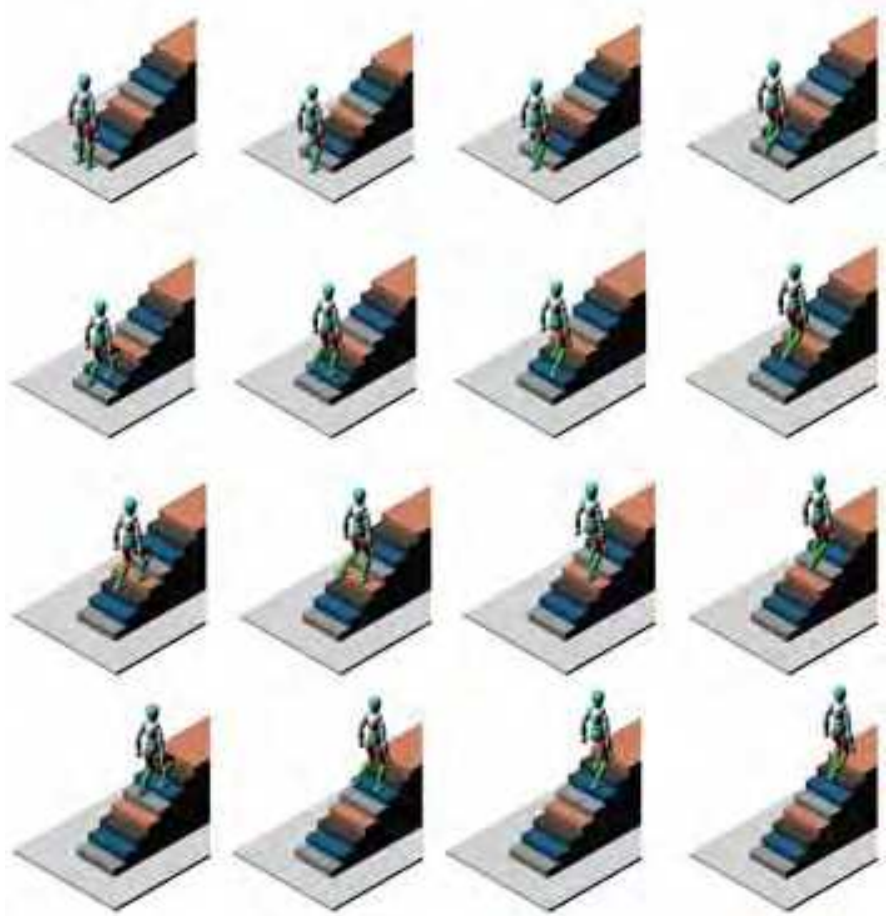

Fig. 19. Motion sequences of a humanoid robot climbing stairs 


\section{References}

C. Zhou .Neuro-fuzzy gait synthesis with reinforcement learning for a biped walking robot, Soft Comput. 4 (2000) 238-250.

G.O.A. ;Zapata\&R.K.H. Galvao, T. Yoneyama, Extraction fuzzy control rules from experimental human operator data, IEEE Trans. Systems Man Cybernet. B 29 (1999) 398-406.

Hirai Kazuo; Hirose Masato \& Haikawa Yuji(1998). The development of honda humanoid robot [A]. Proceedings of 1998 IEEE International Conference on Robotics \& Automation]. Belgium. May 1998:1321-1326

J.H. Park\& K.D. Kim(1998). Biped robot walking using gravity-compensated inverted pendulum mode and computed torque control, Proc. IEEE Internat. Conf. on Robotics and Automation, Leuven, Belgium, 1998. vol.4, pp. 3528-3533

J. Pratt \& G. Pratt. Intuitive control of a planar bipedal walking robot, in: Proc. of IEEE Conf. on Robotics and Automation, 1998, pp. 2014-2021.

J. Yamaguchi; N. Kinoshita; A. Takanish, et al(1996). Development of a dynamic biped walking system for humanoid development of a biped walking robot adapting to the humans' living Goor, Proc. IEEE Internat. Conf. on Robotics and Automation, Minneapolis, MN, 1996, pp. 232-239.

K.Tanie (1999). MITI Humanoid Robotics Project. The 2nd International symposium on humanoid robot, 1999.Tokyo: 71-76

Q. Li; A. Takanish \& I. Kato(1992).Learning control of compensative trunk motion for biped walking robot based on ZMP stability criterion, Proc. IEEE=RSJ Internat. Workshop on Intelligent Robotics and Systems, Raleigh, NC, 1992, pp. 597-603.

Q.Huang; H.Arai \& K.Tanie(1999). A High Stability Smooth Walking Pattern for Biped Robot. IEEE International Conference on Robotics and Automation, 1999,pp:65-71

S.Hashimoto, et al (1998).“ Humanoid Robots in Waseda University -Hadaly-2 and WABIAN" IARP First International Workshop on Humanoid and Human Friendly Robotics , October Japan , 1998 , pp. I-2: 1-10

S. Kajita\& K. Tani(1995)., Experimental study of biped dynamic walking in the linear inverted pendulum mode, Proc. IEEE Internat. Conf. on Robotics and Automation, Nagoya, Japan, 1995, pp. 2885-2891. 


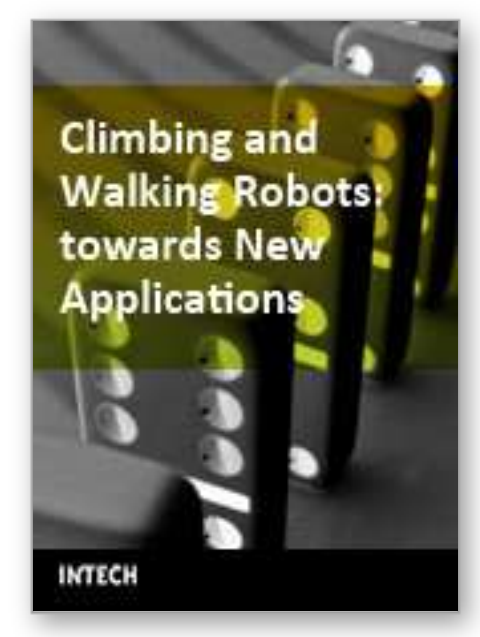

\author{
Climbing and Walking Robots: towards New Applications \\ Edited by Houxiang Zhang
}

ISBN 978-3-902613-16-5

Hard cover, 546 pages

Publisher I-Tech Education and Publishing

Published online 01, October, 2007

Published in print edition October, 2007

With the advancement of technology, new exciting approaches enable us to render mobile robotic systems more versatile, robust and cost-efficient. Some researchers combine climbing and walking techniques with a modular approach, a reconfigurable approach, or a swarm approach to realize novel prototypes as flexible mobile robotic platforms featuring all necessary locomotion capabilities. The purpose of this book is to provide an overview of the latest wide-range achievements in climbing and walking robotic technology to researchers, scientists, and engineers throughout the world. Different aspects including control simulation, locomotion realization, methodology, and system integration are presented from the scientific and from the technical point of view. This book consists of two main parts, one dealing with walking robots, the second with climbing robots. The content is also grouped by theoretical research and applicative realization. Every chapter offers a considerable amount of interesting and useful information.

\title{
How to reference
}

In order to correctly reference this scholarly work, feel free to copy and paste the following:

Xianxin Ke, Jinwu Qian and Zhenbang Gong (2007). Non-Time Reference Gait Planning and Stability Control for Bipedal Walking, Climbing and Walking Robots: towards New Applications, Houxiang Zhang (Ed.), ISBN: 978-3-902613-16-5, InTech, Available from:

http://www.intechopen.com/books/climbing_and_walking_robots_towards_new_applications/nontime_reference_gait_planning_and_stability_control_for_bipedal_walking

\section{INTECH}

open science | open minds

\author{
InTech Europe \\ University Campus STeP Ri \\ Slavka Krautzeka 83/A \\ 51000 Rijeka, Croatia \\ Phone: +385 (51) 770447 \\ Fax: +385 (51) 686166 \\ www.intechopen.com
}

\author{
InTech China \\ Unit 405, Office Block, Hotel Equatorial Shanghai \\ No.65, Yan An Road (West), Shanghai, 200040, China \\ 中国上海市延安西路65号上海国际贵都大饭店办公楼 405 单元 \\ Phone: +86-21-62489820 \\ Fax: +86-21-62489821
}


(C) 2007 The Author(s). Licensee IntechOpen. This chapter is distributed under the terms of the Creative Commons Attribution-NonCommercial-ShareAlike-3.0 License, which permits use, distribution and reproduction for non-commercial purposes, provided the original is properly cited and derivative works building on this content are distributed under the same license. 